\title{
On Lorentz-Minkowski geometry in real inner product spaces
}

\author{
Walter Benz \\ Dedicated to Adriano Barlotti on the occasion of his 80 th birthday, in friendship
}

Let $X$ be a real inner product space of finite or infinite dimension $\geqslant 2$, and let $\varrho \neq 0$ be a fixed real number. The following results will be presented in this note.

A. A surjective mapping $\sigma: X \rightarrow X$ preserving Lorentz-Minkowski distances 0 and $\varrho$ in one direction must be a Lorentz transformation.

B. The causal automorphisms of $X, \operatorname{dim} X \geqslant 3$, are exactly the products $\delta \lambda$, where $\lambda$ is an orthochronous Lorentz transformation and $\delta$ a dilatation $x \rightarrow \alpha x$, $\mathbb{R} \ni \alpha>0$.

C. If $\varrho>0$, there exist $X$ and an injective $\sigma: X \rightarrow X$ preserving Lorentz-Minkowski distance $\varrho$, such that $\sigma$ is not a Lorentz transformation. This result can be extended, mutatis mutandis, to Euclidean and Hyperbolic Geometry.

If $X$ is finite-dimensional, result $\mathrm{A}$ is an immediate consequence of the following theorem of Benz-Lester ([4], [12], [13], [5]).

Theorem 1. Suppose that $X$ is a real inner product space of finite dimension $\geqslant 2$ and that $\varrho \neq 0$ is a fixed real number. If $\sigma: X \rightarrow X$ satisfies

$$
l(x, y)=\varrho \Rightarrow l(\sigma(x), \sigma(y))=\varrho
$$

for all $x, y \in X$, where $l(x, y)$ designates the Lorentz-Minkowski distance of $x, y$, then $\sigma$ must be a Lorentz transformation.

Moreover, if $X$ is finite-dimensional, statement $\mathrm{B}$ is a well-known theorem of Alexandrov-Ovchinnikova-Zeeman ([1], [2], [17], [5]).

It could be possible that Theorem 1 also holds true in the infinite-dimensional case provided that $\varrho<0$. However, a proof, if it exists, is not yet known. Result $\mathrm{C}$ shows that Theorem 1 cannot be extended to the infinite-dimensional case if $\varrho>0$, not even in the injective case. 


\section{Notation}

Let $X$ be a real inner product space of arbitrary finite or infinite dimension $\geqslant 2$, i.e. a real vector space equipped with a fixed inner product

$$
\tau: X \times X \rightarrow \mathbb{R}, \quad \tau(x, y)=: x y,
$$

satisfying $x^{2}:=x x>0$ for all $x \neq 0$ of $X$. Notice that $X$ need not be complete, i.e. that $X$ need not be a real Hilbert space. Take a fixed $t \in X$ with $t^{2}=1$ and define $t^{\perp}:=\{x \in X \mid x t=0\}$. Observe $X=t^{\perp} \oplus \mathbb{R} t$. We hence get the uniquely determined decomposition

$$
x=: \bar{x}+x_{0} t
$$

with $\bar{x} \in t^{\perp}$ and $x_{0} \in \mathbb{R}$ for every $x \in X$. Define

$$
l(x, y):=(\bar{x}-\bar{y})^{2}-\left(x_{0}-y_{0}\right)^{2}
$$

to be the Lorentz-Minkowski distance of $x, y \in X$. The mapping $\lambda: X \rightarrow X$ is called a Lorentz transformation if, and only if,

$$
l(x, y)=l(\lambda(x), \lambda(y))
$$

holds true for all $x, y \in X$.

Remark. It might be noticed that the theory does not seriously depend on the chosen $t$ ([6], p. 229).

The Lorentz transformations as defined before can explicitly be written by means of (proper or improper) Lorentz boosts and orthogonal transformations ([6], p. 221): For $p \in t^{\perp}$ with $p^{2}<1$ and $-1 \neq k \in \mathbb{R}$ with $k^{2} \cdot\left(1-p^{2}\right)=1$ define for all $x \in X$,

$$
A_{p}(x):=x_{0} p+(\bar{x} p) t, \quad B_{p, k}(x):=x+k A_{p}(x)+\frac{k^{2}}{k+1} A_{p}^{2}(x) .
$$

Obviously, $k^{2} \geqslant 1$. The mappings $A_{p}, B_{p, k}$ are linear and $B_{p, k}: X \rightarrow X$ is even bijective. Define also

$$
B_{0,-1}(x):=\bar{x}-x_{0} t
$$

$B_{p, k}$ is called a Lorentz boost, a proper one for $k \geqslant 1$, an improper one for $k \leqslant-1$. All Lorentz transformations $\lambda$ of $X$ are exactly given by

$$
\lambda(x)=\left(B_{p, k} \omega\right)(x)+d
$$

with a boost $B_{p, k}$, an orthogonal and linear mapping $\omega$ from $X$ into $X$ satisfying $\omega(t)=t$, and with an element $d$ of $X$. 
The following theorem was proved by Cacciafesta [9] in the case $\operatorname{dim} X<\infty$, and by Benz [7] in the general case.

Theorem 2. If $\operatorname{dim} X \geqslant 3$ and if $\sigma: X \rightarrow X$ is bijective and satisfies

$$
l(x, y)=0 \Rightarrow l(\sigma(x), \sigma(y))=0
$$

for all $x, y \in X$, then $\sigma$ must be the product of a Lorentz transformation and a dilatation.

Important partial results of Theorem 2 were proved by Alexandrov [1] and by Schröder [15], [16]. Schröder even studied the case of an arbitrary field instead of $\mathbb{R}$.

\section{Proof of result $A$}

Lemma 1. Let $\gamma$ be a real number and $x \neq 0$ be an element of $X$. Then there exist $v \neq 0$ in $X$ and $\alpha$ in $\mathbb{R}$ with $\bar{v}^{2}=v_{0}^{2}$ and

$$
(\bar{x}+\alpha \bar{v})^{2}-\left(x_{0}+\alpha v_{0}\right)^{2}=\gamma .
$$

Proof. Case 1: $x_{0} \neq 0$. Take an element $e$ in $t^{\perp}$ with $e^{2}=1$. Then $\bar{x} e \neq x_{0}$ or $\bar{x} e \neq-x_{0}$. Assume $\bar{x} e \neq \varepsilon x_{0}$ with $\varepsilon \in \mathbb{R}$ and $\varepsilon^{2}=1$. Now put $v:=e+\varepsilon t$ and, by observing $\bar{x} \bar{v} \neq x_{0} v_{0}$,

$$
2 \alpha\left(\bar{x} \bar{v}-x_{0} v_{0}\right):=\gamma+x_{0}^{2}-\bar{x}^{2}
$$

Hence (1) holds true.

Case 2: $x_{0}=0$. Hence $x \neq 0$ implies $\bar{x} \neq 0$. Now put $v:=\bar{x}+\|\bar{x}\| \cdot t$ with $\|z\|:=\sqrt{z^{2}}$ for $z \in X$, and define $\alpha$ by (2). Then also here (1) holds true.

Lemma 2. If $p \neq q$ are elements of $X$ and if $\gamma \in \mathbb{R}$, there exists $r \in X$ satisfying

$$
l(r, p)=\gamma \quad \text { and } \quad l(r, q)=0 .
$$

Proof. Put $x:=q-p$ and take elements $v$ and $\alpha$ according to Lemma 1. Hence $r:=q+\alpha v$ satisfies (3).

Lemma 3. Suppose that $\varrho \neq 0$ is a fixed real number and that $\sigma: X \rightarrow X$ satisfies

$$
l(x, y)=0 \Rightarrow l(\sigma(x), \sigma(y))=0
$$

and

$$
l(x, y)=\varrho \Rightarrow l(\sigma(x), \sigma(y))=\varrho
$$

for all $x, y \in X$. Then $\sigma$ must be injective. 
Proof. If $p \neq q$ are elements of $X$, take, in view of Lemma 2, $r \in X$ with $l(r, p)=\varrho$ and $l(r, q)=0$. Hence, by (4), (5),

$$
l\left(r^{\prime}, p^{\prime}\right)=\varrho \text { and } l\left(r^{\prime}, q^{\prime}\right)=0
$$

where we put $z^{\prime}=\sigma(z)$ for $z \in X$. Now (6) implies $p^{\prime} \neq q^{\prime}$.

If $\operatorname{dim} X<\infty$, result A follows from Theorem 1. Suppose now that $X$ is infinitedimensional and that $\sigma: X \rightarrow X$ is surjective, satisfying (4) and (5) for all $x, y \in X$, where $\varrho \neq 0$ is a fixed real number. Hence, by Lemma 3, $\sigma$ is injective, and thus bijective. Hence, by Theorem 2, there exists a Lorentz transformation $\lambda: X \rightarrow X$ and a real number $k \neq 0$ such that

$$
\sigma(x)=k \cdot \lambda(x)
$$

for all $x \in X$. Now (5) implies

$$
l(x, y)=\varrho \Rightarrow \varrho=l(k \lambda(x), k \lambda(y))
$$

for all $x, y \in X$, i.e. $l(x, y)=\varrho$ implies

$$
\varrho=k^{2} \cdot l(\lambda(x), \lambda(y))=k^{2} \cdot l(x, y)=k^{2} \cdot \varrho .
$$

Hence $k^{2}=1$, in view of $\varrho \neq 0$. If $k=1$, we get $\sigma=\lambda$, and if $k=-1$, we obtain

$$
\sigma(x)=-\lambda(x)
$$

for all $x \in X$. But this is also a Lorentz transformation.

So we have proved

Theorem A. Let $\varrho \neq 0$ be a fixed real number and let $\sigma: X \rightarrow X$ be a surjective mapping satisfying (4) and (5) for all $x, y \in X$. Then there exist a Lorentz boost $B_{p, k}, a$ linear, bijective and orthogonal mapping $\omega: X \rightarrow X$ with $\omega(t)=t$, and an element $d$ of $X$ such that

$$
\sigma(x)=\left(B_{p, k} \omega\right)(x)+d
$$

for all $x \in X$.

Remark. Theorem A holds true, as was shown, for all real inner product spaces $X$ with $\operatorname{dim} X \geqslant 2$. If $X=\mathbb{R}^{2}$, if we put

$$
x y:=x_{1} y_{1}+x_{2} y_{2}
$$

for $x=\left(x_{1}, x_{2}\right), y=\left(y_{1}, y_{2}\right)$ of $X$, and $t:=(1 / \sqrt{2}, 1 / \sqrt{2})$, then

$$
l(x, y)=-2\left(x_{1}-y_{1}\right)\left(x_{2}-y_{2}\right)
$$


for all $x, y \in X$. Let $f$ be a non-continuous bijection of $\mathbb{R}$, for instance $f(0)=1$, $f(1)=0$ and $f(x)=x$ otherwise, then

$$
\sigma\left(x_{1}, x_{2}\right):=\left(f\left(x_{1}\right), x_{2}\right)
$$

is a non-continuous bijection of $X$ satisfying

$$
l(x, y)=0 \Leftrightarrow l(\sigma(x), \sigma(y))=0
$$

for all $x, y \in X$ (Rätz [14]). Hence $\sigma$ cannot be a Lorentz transformation, and it even cannot be a product of a Lorentz transformation and a dilatation. In the case $\operatorname{dim} X \geqslant 2$, the mapping $\sigma(x)=2 x$ is bijective, it satisfies (4), but not (5) for any given $\varrho \neq 0$. So it cannot be a Lorentz transformation.

\section{Causal automorphisms}

Let $x, y$ be elements of $X$. Also in the infinite-dimensional case we put

$$
x \leqslant y
$$

if, and only if, $l(x, y) \leqslant 0$ and $x_{0} \leqslant y_{0}$ hold true. A bijection $\sigma: X \rightarrow X$ is called a causal automorphism if, and only if,

$$
x \leqslant y \Leftrightarrow \sigma(x) \leqslant \sigma(y)
$$

for all $x, y \in X$.

The proof of Proposition 1 is not difficult.

Proposition 1. Let $x, y, z$ be elements of $X$ and let $k$ be a real number. Then the following statements hold true.

(i) $x \leqslant x$,

(ii) $x \leqslant y$ and $y \leqslant x$ imply $x=y$,

(iii) $x \leqslant y$ and $y \leqslant z$ imply $x \leqslant z$,

(iv) $x \leqslant y$ implies $x+z \leqslant y+z$,

(v) $x \leqslant y$ implies $k x \leqslant k y$ for $k \geqslant 0$,

(vi) $x \leqslant y$ implies $k x \geqslant k y$ for $k<0$.

Of course, $x<y$ stands for $x \leqslant y$ and $x \neq y, x \geqslant y$ for $y \leqslant x$, and $x>y$ for $y<x$.

Suppose that $x, y$ are elements of $X$ satisfying $x<y$. Then

$$
[x, y]:=\{z \in X \mid x \leqslant z \leqslant y\}
$$

is called ordered if, and only if, 


$$
u \leqslant v \text { or } v \leqslant u
$$

holds true for all $u, v \in[x, y]$.

Proposition 2. Let $x, y$ be elements of $X$ with $x<y$. Then $l(x, y)=0$ if, and only if, $[x, y]$ is ordered.

Proof. a) Assume $l(x, y)=0$ and $u \in[x, y]$, i.e.

$$
x_{0} \leqslant u_{0} \leqslant y_{0}, \quad\|\bar{u}-\bar{x}\| \leqslant u_{0}-x_{0}, \quad\|\bar{y}-\bar{u}\| \leqslant y_{0}-u_{0} .
$$

$l(x, y)=0$ implies $\|\bar{y}-\bar{x}\|=y_{0}-x_{0}$. Hence

$$
y_{0}-x_{0}=\|\bar{y}-\bar{x}\| \leqslant\|\bar{y}-\bar{u}\|+\|\bar{u}-\bar{x}\| \leqslant y_{0}-x_{0},
$$

and thus $\|\bar{y}-\bar{x}\|=\|\bar{y}-\bar{u}\|+\|\bar{u}-\bar{x}\|$. Since $X$ is strictly convex, $\bar{y}-\bar{u}, \bar{u}-\bar{x}$ must be linearly dependent. Hence there exists $\alpha \in \mathbb{R}$ with

$$
\bar{u}=\bar{x}+\alpha(\bar{y}-\bar{x})
$$

in view of $\bar{x} \neq \bar{y}$; observe that $\bar{x}=\bar{y}$ and $\|\bar{y}-\bar{x}\|=y_{0}-x_{0}$ would imply $x=y$. Now (7), (8) yield

$$
\|\bar{y}-\bar{x}\|=\|\bar{y}-\bar{u}\|+\|\bar{u}-\bar{x}\|=|1-\alpha|\|\bar{y}-\bar{x}\|+|\alpha|\|\bar{y}-\bar{x}\|,
$$

i.e. $1=|1-\alpha|+|\alpha|$, i.e. $0 \leqslant \alpha \leqslant 1$. Hence, with $\xi:=y_{0}-x_{0}$,

$$
\xi=(1-\alpha) \xi+\alpha \xi=\|\bar{y}-\bar{u}\|+\|\bar{u}-\bar{x}\| \leqslant\left(y_{0}-u_{0}\right)+\left(u_{0}-x_{0}\right)=\xi
$$

i.e. $\|\bar{y}-\bar{u}\|=y_{0}-u_{0},\|\bar{u}-\bar{x}\|=u_{0}-x_{0}$, i.e. by (8),

$$
u=x+\alpha(y-x)
$$

Similarly, $v \in[x, y]$ implies

$$
v=x+\beta(y-x), \quad 0 \leqslant \beta \leqslant 1 .
$$

Hence $u \leqslant v$ for $\alpha \leqslant \beta$, and $v \leqslant u$ for $\beta \leqslant \alpha$.

b) Assume that $[x, y]$ is ordered and that $l(x, y) \neq 0$. Hence, by $x<y$, we obtain $l(x, y)<0$ and $x_{0} \leqslant y_{0}$, i.e.

$$
(\bar{y}-\bar{x})^{2}<\left(y_{0}-x_{0}\right)^{2} \text { and } x_{0}<y_{0} .
$$

Choose $e \in t^{\perp}$ with $e^{2}=1$ and $\varepsilon \in \mathbb{R}$ with

$$
0<2 \varepsilon<\left(y_{0}-x_{0}\right)-\|\bar{y}-\bar{x}\|
$$


and put

$$
u:=\frac{x+y}{2}, \quad v:=\frac{x+y}{2}+\varepsilon e .
$$

Observe $u_{0}=v_{0}$ and $\bar{v}-\bar{u}=\varepsilon e$, i.e. $l(u, v)=\varepsilon^{2}>0$, i.e.

$$
u \nless v \text { and } v \nless u \text {. }
$$

Moreover,

$$
u, v \in[x, y] .
$$

In order to prove (11), we observe, first of all,

$$
x_{0} \leqslant u_{0} \leqslant y_{0} \text { and } x_{0} \leqslant v_{0} \leqslant y_{0},
$$

by $u_{0}=v_{0}=\frac{1}{2}\left(x_{0}+y_{0}\right)$. Secondly,

$$
l(x, u)=\frac{1}{4} l(x, y)=l(u, y)
$$

i.e. $l(x, u)=l(u, y)<0$. The triangle inequality yields

$$
\left\|\frac{\bar{y}-\bar{x}}{2} \pm \varepsilon e\right\| \leqslant\left\|\frac{\bar{y}-\bar{x}}{2}\right\|+\varepsilon,
$$

i.e. by (9),

$$
\left\|\frac{\bar{y}-\bar{x}}{2} \pm \varepsilon e\right\|<\frac{y_{0}-x_{0}}{2} .
$$

Hence

$$
\left(\frac{\bar{y}-\bar{x}}{2} \pm \varepsilon e\right)^{2}<\left(\frac{y_{0}-x_{0}}{2}\right)^{2}
$$

i.e. $l(x, v)$ and $l(v, y)$ are negative. Because of $(10),(11),[x, y]$ is not ordered, a contradiction. Hence $l(x, y)=0$.

A Lorentz transformation $\lambda$ of $X$ is called orthochronous if, and only if, it is also a causal automorphism.

Proposition 3. The orthochronous Lorentz transformations $\lambda$ are exactly given by all mappings 


$$
\lambda(x)=\left(B_{p, k} \omega\right)(x)+d
$$

with $\omega: X \rightarrow X$ linear, orthogonal, bijective, $\omega(t)=t, d \in X$, and $k \geqslant 1$.

Proof. a) Let $\lambda$ be an arbitrary orthochronous Lorentz transformation, say

$$
\lambda(x)=\left(B_{p, k} \omega\right)(x)+d .
$$

Since $\lambda$ is bijective, also $\omega: X \rightarrow X$ must be bijective. Moreover, $0 \leqslant t$ implies $\lambda(0) \leqslant \lambda(t)$, i.e.

$$
d \leqslant k t+k p+d
$$

i.e. $0 \leqslant k p+k t$, i.e. $0 \leqslant k$, i.e. $1 \leqslant k$, in view of $k^{2} \geqslant 1$.

b) Let $\lambda$ be a mapping (12) with proper $B_{p, k}$ and bijective $\omega$ satisfying $\omega(t)=t$. We then have to prove

$$
a \leqslant b \Leftrightarrow \lambda(a) \leqslant \lambda(b)
$$

for all $a, b \in X$. This is clear for $\lambda(x)=x+d$, in view of Proposition 1 (iv). It is also clear for $\lambda(x)=\omega(x)$ because of

$$
\begin{aligned}
0 \geqslant l(a, b) & =l(\lambda(a), \lambda(b)), \\
\omega\left(\bar{x}+x_{0} t\right) & =\omega(\bar{x})+x_{0} t, \\
\omega(\bar{x}) t & =\omega(\bar{x}) \omega(t)=\bar{x} t=0,
\end{aligned}
$$

i.e. $\overline{\omega(x)}=\omega(\bar{x})$, and on account of the fact that $\omega^{-1}$ is linear and orthogonal as well, satisfying $\omega^{-1}(t)=t$.

Finally, we consider the case $\lambda(x)=B_{p, k}(x)$ with $k \geqslant 1$. Since $B_{p, k}^{-1}=B_{-p, k}$ we only have to prove

$$
a \leqslant b \Rightarrow \lambda(a) \leqslant \lambda(b)
$$

i.e. $0 \leqslant b-a \Rightarrow 0 \leqslant \lambda(b)-\lambda(a)=\lambda(b-a)$, i.e.

$$
0 \leqslant x \Rightarrow 0 \leqslant \lambda(x) \text {. }
$$

Again, $0 \geqslant l(0, x)=l(\lambda(0), \lambda(x))=l(0, \lambda(x))$. Since $z_{0}=z t$ for all $z \in X$, we get

$$
\left[B_{p, k}(x)\right]_{0}=x_{0}(1+k(k-1))+k \bar{x} p=: R .
$$

It remains to prove $R \geqslant 0$ in the case $0 \leqslant x_{0}$ and $\bar{x}^{2}-x_{0}^{2}=l(0, x) \leqslant 0$. If $\bar{x} p \geqslant 0$, we get $R \geqslant 0$ since $k \geqslant 1$. If $\bar{x} p<0$, we observe

$$
(\bar{x} p)^{2} \leqslant \bar{x}^{2} p^{2} \leqslant x_{0}^{2} \cdot 1=x_{0}^{2}
$$


i.e. $-\bar{x} p=|\bar{x} p| \leqslant x_{0}$, i.e. $-x_{0} \leqslant \bar{x} p$, i.e.

$$
R \geqslant x_{0}(1+k(k-1))-k x_{0}=x_{0}(k-1)^{2} \geqslant 0 .
$$

Theorem B. The causal automorphisms of $X, \operatorname{dim} X \geqslant 3$, are exactly given by all mappings

$$
\lambda(x)=\gamma \cdot\left(B_{p, k} \omega\right)(x)+d,
$$

where $\gamma>0$ is a real number, $B_{p, k}$ a proper Lorentz boost, $\omega$ a linear, orthogonal, bijective mapping of $X$ with $\omega(t)=t, d$ an element of $X$.

Proof. Observe that $\mu(x):=\gamma x$ defines a causal automorphism for a real constant $\gamma>0$. Hence, by Proposition 3, (13) must be a causal automorphism as well.

Suppose now that $\lambda: X \rightarrow X$ is an arbitrary causal automorphism. If $x \neq y$ are elements of $X$ with $l(x, y)=0$, we may assume $x_{0} \leqslant y_{0}$ without loss of generality, and hence $x<y$. Thus, by Proposition $2,[x, y]$ is ordered. Since $\lambda$ is a causal automorphism, also $[\lambda(x), \lambda(y)]$ must be ordered and $\lambda(x)<\lambda(y)$ holds true. Hence, by Proposition $2, l(\lambda(x), \lambda(y))=0$. Now Theorem 2 implies that

$$
\lambda(x)=m \cdot \lambda_{1}(x)
$$

for all $x \in X$, where $\lambda_{1}$ is a Lorentz transformation and $m \neq 0$ a real constant. We may assume $m>0$ without loss of generality, since otherwise we would consider

$$
\lambda(x)=(-m) \cdot\left(-\lambda_{1}(x)\right)
$$

by observing that also $x \rightarrow-\lambda_{1}(x)$ is a Lorentz transformation. Hence

$$
x \rightarrow \frac{1}{m} \lambda(x)
$$

is a causal automorphism, and thus, by (14), $\lambda_{1}$ is an orthochronous Lorentz transformation. In view of Proposition 3, we hence get (13) with the properties described in Theorem B.

\section{Proof of result $\mathrm{C}$}

Let $B$ be a set with $\operatorname{card}(B) \geqslant \aleph:=\operatorname{card}(\mathbb{R})$ and define $X$ to be the set of all functions

$$
x: B \rightarrow \mathbb{R}
$$

such that $\{b \in B \mid x(b) \neq 0\}$ is finite. We shall write $x$ also in the form

$$
x=\sum_{b \in B} x(b) \cdot b
$$


According to this notation, the element $b \in B$ is equal to the element $x$ of $X$ with $x(b)=1$ and $x\left(b^{\prime}\right)=0$ for all $b^{\prime} \neq b$ in $B$.

Define $x+y, \lambda x, x y$ for $x, y \in X, \lambda \in \mathbb{R}$, by means of

$$
x+y:=\sum_{b \in B}[x(b)+y(b)] b, \quad \lambda x:=\sum_{b \in B}[\lambda \cdot x(b)] b
$$

and $x y=\sum_{b \in B} x(b) y(b)$. Then $X$ is a real inner product space of dimension card $(B)$ with basis $B$. If $\left\{b_{1}, \ldots, b_{n}\right\}$ is a finite subset of $B$, then there exist exactly

$$
\aleph \cdot \operatorname{card}(B)=\operatorname{card}(B)
$$

elements of the form $r_{1} b_{1}+\cdots+r_{n} b_{n}$ with real $r_{i}, i=1, \ldots, n$. Since the set of all finite subsets of $B$ has also cardinality $\operatorname{card}(B)$, we get

$$
\operatorname{card}(X)=\operatorname{card}(B) .
$$

Take a fixed $t \in B$. Hence $t^{2}=1$ and

$$
\operatorname{card}(X)=\operatorname{card}(B \backslash\{t\}) .
$$

Therefore there exists a bijection $\mu: X \rightarrow B \backslash\{t\}$. Suppose that $\varrho>0$ is a fixed real number and define

$$
\sigma(x):=\sqrt{\frac{\varrho}{2}} \mu(x) .
$$

Hence $\sigma: X \rightarrow X$ must be injective. Writing again

$$
x=: \bar{x}+x_{0} t, \quad \bar{x} \in t^{\perp}, x_{0} \in \mathbb{R},
$$

for $x \in X$, let $x, y$ be elements of $X$ with

$$
\varrho=l(x, y)=(\bar{x}-\bar{y})^{2}-\left(x_{0}-y_{0}\right)^{2} .
$$

In view of $x \neq y$, the elements $b_{1}:=\mu(x), b_{2}:=\mu(y)$ are distinct. Observe $b_{1}, b_{2} \in$ $B \backslash\{t\}$, i.e. $b_{1}, b_{2} \in t^{\perp}$. Thus

$$
l(\sigma(x), \sigma(y))=\left(\sqrt{\frac{\varrho}{2}} b_{1}-\sqrt{\frac{\varrho}{2}} b_{2}\right)^{2}=\varrho .
$$

Of course, $\sigma$ cannot be a Lorentz transformation, since $l(\sigma(x), \sigma(y))=\varrho$ holds true for all distinct elements $x, y$ of $X$.

Remark. The method followed in this section can be applied to Euclidean and Hyper- 
bolic Geometry in order to find injective mappings $\sigma: X \rightarrow X$ which are not distance preserving, but which preserve a fixed distance $\varrho>0$. Non-injective mappings leaving invariant a fixed distance $\varrho$, but not all other distances, were given by Beckman and Quarles [3] in the euclidean case, and by Benz [8] in the hyperbolic case. In this connection observe theorems of Beckman, Quarles [3], Farrahi [10], Kuz'minyh [11], in which, in the finite-dimensional case, distance preserving mappings are characterized by the invariance of one single distance $\varrho>0$.

Let $B$ be a set with a cardinality $\geqslant \aleph$, and consider the real inner product space $X$ as defined at the beginning of this section. In view of $\operatorname{card}(B)=\operatorname{card}(X)$, there exists a bijection

$$
\mu: X \rightarrow B
$$

In the euclidean case with the distance notion

$$
d(x, y):=\|x-y\|
$$

for all $x, y \in X$, we define for a fixed $\varrho>0$,

$$
\sigma(x):=\frac{\varrho}{\sqrt{2}} \mu(x)
$$

Hence $d(\sigma(x), \sigma(y))=\sqrt{(\sigma(x)-\sigma(y))^{2}}=\varrho$ for $x \neq y$ with $x, y \in X$. So every distance $\neq 0$ goes over in distance $\varrho$.

In the hyperbolic case with the distance notion

$$
h(x, y) \geqslant 0 \text { and } \cosh h(x, y):=\sqrt{1+x^{2}} \sqrt{1+y^{2}}-x y
$$

we define for a fixed $\varrho>0$,

$$
\sigma(x):=\sqrt{2} \sinh \frac{\varrho}{2} \cdot \mu(x)
$$

Hence $\cosh h(\sigma(x), \sigma(y))=1+2 \sinh ^{2} \frac{\varrho}{2}=\cosh \varrho$, i.e.

$$
h(\sigma(x), \sigma(y))=\varrho
$$

for every $x \neq y$ with $x, y \in X$. So also here every distance $\neq 0$ goes over in distance $\varrho$.

\section{References}

[1] A. D. Alexandrov, Seminar report. Uspehi Mat. Nauk. 5 (1950), no. 3 (37), 187.

[2] A. D. Alexandrov, V. V. Ovchinnikova, Notes on the foundations of relativity theory. Vestnik Leningrad. Univ. 11, 95 (1953). 
[3] F. S. Beckman, D. A. Quarles, Jr., On isometries of Euclidean spaces. Proc. Amer. Math. Soc. 4 (1953), 810-815. MR 15,335a Zbl 0052.18204

[4] W. Benz, Eine Beckman-Quarles-Charakterisierung der Lorentztransformationen des $\mathbb{R}^{n}$. Arch. Math. (Basel) 34 (1980), 550-559. MR 82h:51022 Zbl 0446.51015

[5] W. Benz, Geometrische Transformationen. Bibliographisches Institut, Mannheim 1992. MR 93i:51002 Zbl 0754.51005

[6] W. Benz, Lorentz-Minkowski distances in Hilbert spaces. Geom. Dedicata 81 (2000), 219-230. MR 2001e:46032 Zbl 0959.51013

[7] W. Benz, Lie sphere geometry in Hilbert spaces. Results Math. 40 (2001), 9-36. MR 2003b:51007 Zbl 0995.51003

[8] W. Benz, Mappings preserving two hyperbolic distances. J. Geom. 70 (2001), 8-16. MR 2002g:51017 Zbl 0988.51015

[9] F. Cacciafesta, An observation about a theorem of A. D. Alexandrov concerning Lorentz transformations. J. Geom. 18 (1982), 5-8. MR 83h:51026 Zbl 0485.51015

[10] B. Farrahi, A characterization of isometries of absolute planes. Resultate Math. 4 (1981), 34-38. MR 82i:51022 Zbl 0472.51009

[11] A. V. Kuz'minyh, Mappings preserving the distance 1. Sibirsk. Mat. Zh. 20 (1979), 597-602. MR 80h:51016 Zbl 0427.51008

[12] J. A. Lester, The Beckman-Quarles theorem in Minkowski space for a spacelike squaredistance. Arch. Math. (Basel) 37 (1981), 561-568. MR 83e:51008 Zbl 0457.51027

[13] J. A. Lester, Distance preserving transformations. In: Handbook of incidence geometry, 921-944, North-Holland 1995. MR 96j:51019 Zbl 0826.51010

[14] J. Rätz, On light-cone-preserving mappings of the plane. In: General inequalities, 3 (Oberwolfach, 1981), volume 64 of Internat. Schriftenreihe Numer. Math., 349-367, Birkhäuser 1983. MR 86e:51025 Zbl 0527.51006

[15] E. M. Schröder, Zur Kennzeichnung distanztreuer Abbildungen in nichteuklidischen Räumen. J. Geom. 15 (1980), 108-118. MR 82h:51025 Zbl 0463.51015

[16] E. M. Schröder, Ein einfacher Beweis des Satzes von Alexandrov-Lester. J. Geom. 37 (1990), 153-158. MR 9lc:51019 Zbl 0704.51010

[17] E. C. Zeeman, Causality implies the Lorentz group. J. Mathematical Phys. 5 (1964), 490-493. MR 28 \#5785 Zbl 0133.23205

Received 8 October, 2002

W. Benz, Mathematisches Seminar der Universität, Bundesstr. 55, 20146 Hamburg, Germany Email: benz@math.uni-hamburg.de 